\title{
Postoperative symbiotic in patients with head and neck cancer: a double-blind randomised trial
}

\author{
Priscilla C. Lages ${ }^{1 *}$, Simone V. Generoso ${ }^{1}$ and Maria Isabel T. D. Correia ${ }^{2}$ \\ ${ }^{1}$ Department of Nutrition, Universidade Federal de Minas Gerais, Minas Gerais, 30130-100, Brazil \\ ${ }^{2}$ Surgery Department, Universidade Federal de Minas Gerais, Minas Gerais, 30130-100, Brazil \\ (Submitted 11 July 2017 - Final revision received 30 October 2017 - Accepted 8 November 2017-First published online 26 December 2017)
}

\section{Abstract}

Studies on the 'gut origin of sepsis' have suggested that stressful insults, such as surgery, can affect intestinal permeability, leading to bacterial translocation. Symbiotics have been reported to be able to improve gut permeability and modulate the immunologic system, thereby decreasing postoperative complications. Therefore we aimed to evaluate the postoperative use of symbiotics in head and neck cancer surgical patients for intestinal function and permeability, as well as the postoperative outcomes. Patients were double-blind randomised into the symbiotic ( $n$ 18) or the control group ( $n$ 18). Samples were administered twice a day by nasoenteric tube, starting on the 1st postoperative day until the 5 th to 7 th day, and comprised $10^{9}$ colony-forming units $/ \mathrm{ml}$ each of Lactobacillus paracasei, L. rhamnosus, L. acidophilus, and Bifidobacterium lactis plus $6 \mathrm{~g}$ of fructo-oligosaccharides, or a placebo ( $6 \mathrm{~g}$ of maltodextrin). Intestinal function (day of first evacuation, total stool episodes, stool consistency, gastrointestinal tract symptoms and gut permeability by diamine oxidase (DAO) enzyme) and postoperative complications (infectious and non-infectious) were assessed. Results of comparison of the pre- and postoperative periods showed that the groups were similar for all outcome variables. In all, twelve patients had complications in the symbiotic group $v$. nine in the control group $(P>0.05$ ), and the preoperative-postoperative DAO activity ranged from 28.5 (sD 15.4 ) to 32.7 (sD 11.0 ) $\mathrm{ng} / \mathrm{ml}$ in the symbiotic group and 35.2 (sD $17 \cdot 7$ ) to $34 \cdot 1$ (sD $12 \cdot 0) \mathrm{ng} / \mathrm{ml}$ in the control group $(P>0 \cdot 05)$. In conclusion, postoperative symbiotics did not impact on intestinal function and postoperative outcomes of head and neck surgical patients.

\section{Key words: Symbiotics: Head and neck cancer: Surgery: Intestinal function: Nutrition therapy}

Symbiotics have been advocated as being beneficial to patients undergoing surgery. Postoperative complications are major concerns that directly affect the clinical evolution and hospital costs of surgical patients. The patient's nutritional status and the 'gut origin of sepsis' theory have been suggested to explain these complications as the result of bacterial translocation (BT), whereby the altered intestinal permeability facilitates the passage of indigenous microbiota to mesenteric lymph nodes and the systemic circulation $^{(1)}$. Undernutrition, a condition widely present among hospitalised patients, further impairs the immunologic/inflammatory response, thus increasing the risk of infections ${ }^{(2)}$.

Symbiotics, 'a mixture of probiotics and prebiotics that beneficially affects the host by improving the survival and implantation of live microbial dietary supplements in the gastrointestinal tract, by selectively stimulating the growth and/ or by activating the metabolism of one or a limited number of health-promoting bacteria, and thus improving host welfare, ${ }^{\text {(3) }}$, have been reported to affect intestinal function and permeability $^{(4)}$. The mechanisms of action can be related to the modulation of the intestinal barrier function, the production of cytokines and immunoglobulins with anti-inflammatory properties, and the capacity to compete against pathogenic bacteria and inhibit their adherence, thus reducing gut permeability and decreasing BT.

Beneficial effects of symbiotics in surgical patients have been shown for over a decade, both pre- and postoperatively, in patients undergoing laparotomy, liver transplantation and resection, colectomy, esophagectomy, pancreatoduodenectomy or other pancreas ressection ${ }^{(5-11)}$. Rayes et al. reported that the use of symbiotics was associated with decreased infection and complication rates, less use of antibiotics and shorter length of hospital stay ${ }^{(5-7)}$. However, other authors have not shown the same positive effects ${ }^{(12,13)}$ and, to our knowledge, no study has assessed the use of symbiotics in head and neck cancer patients.

It was our goal to evaluate the postoperative use of symbiotics in non-abdominal surgical patients (head and neck cancer patients) for intestinal function, intestinal permeability and postoperative outcomes. Thereby, diamine oxidase (DAO) enzyme was chosen for intestinal permeability assessment, as it has a relationship with intestinal maturity and integrity and can

Abbreviations: BT, bacterial translocation; DAO, diamine oxidase.

* Corresponding author: P. C. Lages, fax +55 313409 8028, email priscillaceci@gmail.com 
be considered a regulatory enzyme for intestinal mucosal proliferation $^{(14,15)}$

\section{Methods}

This double-blind randomised controlled trial (RCT) was conducted at the Alfa Institute of Gastroenterology, Hospital das Clínicas da Universidade Federal de Minas Gerais, Belo Horizonte, Minas Gerais, Brazil. Patients were assessed for eligibility if they met the inclusion criteria: older than 18 years, undergoing elective head and neck cancer operations, and having access to the gastrointestinal tract after surgery. Exclusion criteria included the following: presence of inflammatory bowel disease, liver disease or other cancers; undergoing neoadjuvant chemotherapy; use of antibiotics or probiotics 1 month before the trial; and no access to the gastrointestinal tract after surgery. All patients gave written informed consent to enter the study, which was approved by the university ethics committee (CAAE 24375713.0.0000.5149) and registered at Clinical Trials (NCT02654652).

Patients were initially evaluated for baseline characteristics (age, sex, time since diagnosis, $T$ staging of the tumour ${ }^{(16)}$ and tumour localisation) and for nutritional parameters (weight, subjective global nutritional assessment ${ }^{(17)}$ and hand grip strength $\left.^{(18)}\right)$. Patients were allocated by block randomisation into (A) treatment or (B) placebo groups. Patients and the research team were blinded to the interventions. After the operation, patients started receiving $6 \mathrm{~g}$ of treatment samples $\left(10^{9}\right.$ colonyforming units/ml each of Lactobacillus paracasei LPC-31, L. rhamnosus HNOOl, L. acidophilus NCFM, and Bifidobacterium lactis HN019 plus $6 \mathrm{~g}$ of fructo-oligosaccharides) or placebo samples (maltodextrin) packed in identical sachets (Invictus ${ }^{\circledR}$ Farmanutrition FQM Group), on the first postoperative day. The sachets were similarly diluted in $20 \mathrm{ml}$ of water and administered twice a day, for at least $5 \mathrm{~d}$ and up to $7 \mathrm{~d}$, through a nasoenteric catheter placed intraoperatively. Patients in both groups also received enteral nutritional therapy with a standard polymeric formula $(4 \mathrm{~kJ} / \mathrm{ml}(1 \mathrm{kcal} / \mathrm{ml}), 15 \%$ protein, $55 \%$ carbohydrate, $30 \%$ lipids and $220 \mathrm{mOsm} / \mathrm{l}$ ) on the 1 st postoperative day, starting with $40 \%$ of the estimated energetic goal (calculated by using $30 \mathrm{kcal} / \mathrm{kg}$ per $\mathrm{d}$, as standardised by the hospital nutrition team) and continuing until $100 \%$ of the energetic requirements were met on the third postoperative day.

\section{Outcomes}

The primary outcomes were intestinal function and permeability. Intestinal functional comprised the day of first evacuation, the number of total stool episodes within intervention days, stool consistency and gastrointestinal tract adverse symptoms and signs, such as flatulence, abdominal bloating, and cramps. Stool consistency and frequency data were collected daily questioning the patient, and the Bristol stool form scale ${ }^{(19)}$ was used to score the data. Gastrointestinal adverse effects were considered as the total number of symptoms reported by patients throughout the intervention days.

Secondary outcomes were postoperative complications such as wound infection, salivary fistula, pneumonia indicated by pulmonary infiltrates on chest X-ray associated with fever and positive culture results from sputum, bacteraemia with fever and positive blood culture results, sepsis identified as low blood pressure associated with fever and positive blood culture results, other infections with a temperature higher than $38.5^{\circ} \mathrm{C}$ treated with antibiotics, and haemodynamic instability with use of vasopressors ${ }^{(20)}$. Length of hospital stay, calculated from admission day to discharge day; mortality within the first $30 \mathrm{~d}$ after surgery; and length of antibiotic therapy were also collected.

\section{Laboratory data}

Blood samples were drawn preoperatively and at the end of the intervention period to assess TNF- $\alpha$ cytokine levels, C-reactive protein (CRP) levels, global leucocyte count, and intestinal permeability by DAO.

All blood samples were centrifuged at $1000 \boldsymbol{g}$ for $10 \mathrm{~min}$ and the serum was stored at $-80^{\circ} \mathrm{C}$ until analysis. Serum TNF- $\alpha$ cytokine concentrations were measured by a high-sensitivity sandwich ELISA kit (DY210 Lot 1325880), in accordance with the manufacturer's specifications ( $\& \& D$ Systems $\left.{ }^{\circledR}\right)$. The serum DAO enzyme concentration was also determined by a sandwich ELISA kit (SEA656Hu), in accordance with the manufacturer's specifications (Cloud-Clone Corporation ${ }^{\circledR}$ ). Serum CRP and leucocyte levels were analysed by the hospital laboratory and recorded in the medical records.

\section{Statistical analysis and sample size calculation}

The sample size was calculated on the basis of an expected $30 \%$ increase in DAO concentration after intervention, according to the data of Kamiya et al. ${ }^{(21)}$ from a randomised trial. Adopting the criteria of Armitage \& Berry $^{(22)}(5 \%$ significance and power of $80 \%$ ), we calculated the required sample size to be eighteen participants in each group.

Group comparisons were performed by using the Student's $t$ test and the Mann-Whitney $U$ test (expressed as means and standard deviations and medians and ranges) for parametric and non-parametric data, respectively, and the $\chi^{2}$ test for proportions. Between-treatment effects were evaluated with the Student's paired $t$ test and the Wilcoxon test for parametric and non-parametric variables, respectively. ANCOVA was used to compare median values between groups postoperatively, after adjusting for preoperative median values. A $P$ value of $<0.05$ was considered statistically significant. Statistical analysis was performed with SPSS software, version 19.0.

\section{Results}

In all, forty-six patients were assessed for entry into the study from October 2014 until April 2016. Six were ineligible: one with oesophageal cancer, one with cirrhosis, and four because they had inoperable cancers. A total of forty were randomised (nineteen to the symbiotic group and twenty-one to the control group), as depicted in Fig. 1.

Groups were homogeneous in terms of baseline characteristics and nutritional parameters (Table 1). Nutritional therapy was similarly provided in both groups. Patients received a mean 
Fig. 1. Trial flow diagram

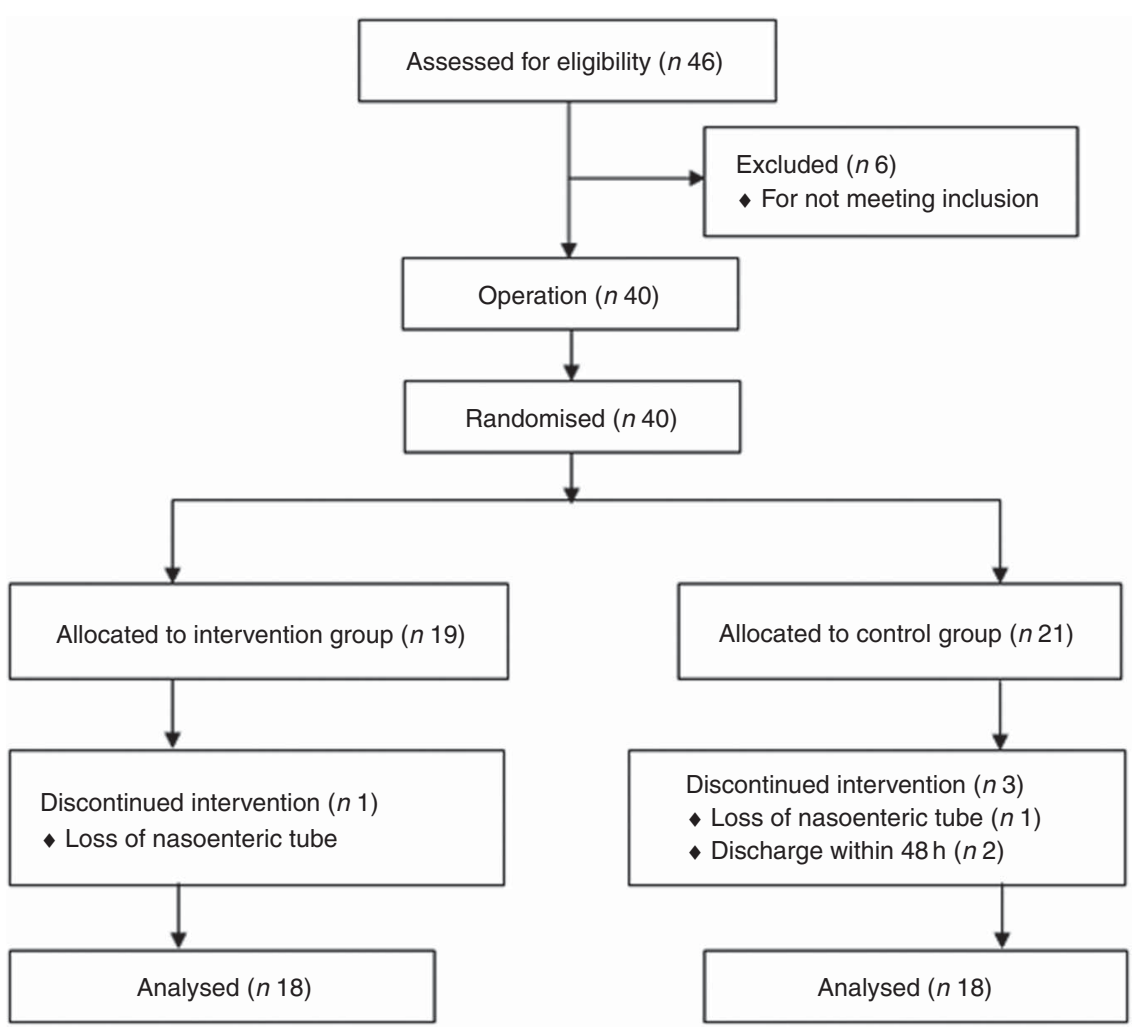

Table 1. Baseline characteristics and nutritional parameters

(Mean values and standard deviations; numbers and ranges)

\begin{tabular}{|c|c|c|c|}
\hline & Symbiotic ( $n$ 18) & Placebo ( $n$ 18) & $P$ \\
\hline Age (years) & & & NS \\
\hline Mean & 59 & 62 & \\
\hline SD & 9 & 13 & \\
\hline Sex, male/female & $13 / 5$ & $16 / 2$ & NS \\
\hline Time since diagnosis (months) & & & NS \\
\hline$n$ & 3 & 3 & \\
\hline Range & $1-24$ & $1-16$ & \\
\hline$T$ stage of tumour & & & NS \\
\hline $\mathrm{T} 2$ & 2 & 4 & \\
\hline T3 & 2 & 1 & \\
\hline $\mathrm{T} 4$ & 14 & 13 & \\
\hline Tumour localisation & & & NS \\
\hline Mouth & 12 & 10 & \\
\hline Laryngeal & 5 & 5 & \\
\hline Pharyngeal & 0 & 3 & \\
\hline Lips & 1 & 0 & \\
\hline Nutritional status by SGA & & & NS \\
\hline Nourished & 6 & 8 & \\
\hline Malnourished & 12 & 10 & \\
\hline Weight (kg) & & & NS \\
\hline Mean & 59.5 & $67 \cdot 0$ & \\
\hline SD & $11 \cdot 7$ & $20 \cdot 0$ & \\
\hline Hand grip strength (kg) & & & NS \\
\hline Mean & $32 \cdot 9$ & $36 \cdot 8$ & \\
\hline SD & $9 \cdot 3$ & 11.4 & \\
\hline
\end{tabular}

SGA, subjective global assessment.

of $4958(\mathrm{sD} 3531) \mathrm{kJ} / \mathrm{d}(1185.3(\mathrm{sD} \quad 844.0) \mathrm{kcal} / \mathrm{d})$ and 43 (sD 15.6$) \mathrm{g} / \mathrm{d}$ of protein, which represents $<70 \%$ of mean nutritional requirements.

Table 2. Intestinal function (Medians and ranges)

\begin{tabular}{|c|c|c|c|c|c|}
\hline & \multicolumn{2}{|c|}{ Symbiotic $(n 18)$} & \multicolumn{2}{|c|}{ Placebo (n 18) } & \multirow[b]{2}{*}{$P$} \\
\hline & Median & Range & Median & Range & \\
\hline Total stool episodes & 4.5 & $2-32$ & 3.5 & $1-11$ & NS \\
\hline Day of first evacuation & 2.5 & $1-7$ & 3.5 & $1-5$ & NS \\
\hline Gastrointestinal symptoms & & & & & NS \\
\hline Flatulence & \multicolumn{2}{|c|}{5} & \multicolumn{2}{|c|}{5} & \\
\hline Abdominal bloating & \multicolumn{2}{|c|}{1} & \multicolumn{2}{|c|}{3} & \\
\hline Abdominal cramps & \multicolumn{2}{|c|}{2} & \multicolumn{2}{|c|}{2} & \\
\hline
\end{tabular}

Both groups presented with similar intestinal function after the intervention (Table 2). Fig. 2 contains data on stool consistency according to the Bristol stool form scale. Intestinal permeability by DAO activity is depicted in Table 3 .

The number of patients with postoperative complications was similar in both groups $(P>0.05)$, as shown in Table 4. There was a higher incidence of fistula in the symbiotic group ( $n$ 8) than in the control group $(n 2 ; P<0.05)$. However, of the eight patients who had fistula in the symbiotic group, three were diagnosed within the first $48 \mathrm{~h}$ after surgery.

A total of eleven patients in the symbiotic and eight in the control group used antibiotics for therapeutic reasons $(P>0.05)$. Patients in the symbiotic group had a median of 10.5 (range 3-90) d of hospitalisation compared with 9.0 (range $5-21$ ) d in the control group $(P>0.05)$. Mortality was null within $30 \mathrm{~d}$ of discharge.

Inflammatory markers were also similar in both groups. TNF- $\alpha$ cytokine concentrations had mean values in the symbiotic group ranging from $16.4(\mathrm{sD} 4.3) \mathrm{pg} / \mathrm{ml}$ in the 


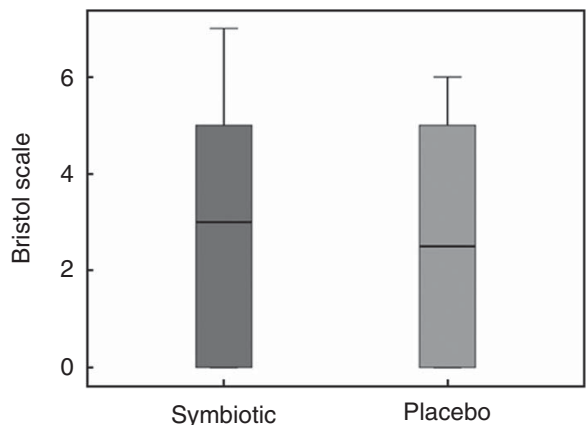

Fig. 2. Stool consistency by the Bristol Stool Scale, a visual scale that categorises stool into seven different consistencies, representing dried stool (1) to liquid stool (7); 0 represents no evacuation at all within the intervention period. Non-significant statistical results were found.

Table 3. Intestinal permeability

(Mean values and standard deviations)

\begin{tabular}{lcccccc}
\hline & \multicolumn{2}{c}{ Symbiotic $(n$ 18) } & & \multicolumn{2}{c}{ Placebo $(n$ 18) } \\
\cline { 2 - 3 } DAO values $(\mathrm{ng} / \mathrm{ml})$ & Mean & SD & & Mean & SD \\
\hline Preoperative & 28.5 & 15.4 & & 35.2 & 17.7 \\
Postoperative & 32.7 & 11.0 & & 34.1 & 12.0 \\
\hline
\end{tabular}

DAO, diamine oxidase.

* Statistical results were non-significant between pre- and postoperative periods and between symbiotic and placebo groups.

Table 4. Postoperative complications

\begin{tabular}{lccc}
\hline & Symbiotic & Placebo & $P$ \\
\hline Patients with complications & 12 & 9 & NS \\
Patients with infection & 11 & 7 & NS \\
No. of infections & 12 & 9 & NS \\
Wound & 3 & 4 & NS \\
Pneumonia & 1 & 2 & NS \\
Bacteraemia & 0 & 1 & NS \\
Fistula & 8 & 2 & $<0.05$ \\
Wound dehiscence & 3 & 2 & NS \\
Haemodynamic instability & 0 & 2 & NS \\
Blood transfusion & 1 & 0 & NS \\
Deaths & 0 & 0 & \\
\hline
\end{tabular}

preoperative period to 14.9 (SD 4.7$) \mathrm{pg} / \mathrm{ml}$ in the postoperative period and in the control group ranging from $15 \cdot 2(\mathrm{SD} 4 \cdot 1) \mathrm{pg} / \mathrm{ml}$ preoperatively to 15.4 (SD 4.7 ) $\mathrm{pg} / \mathrm{ml}$ postoperatively, without statistical significance between groups or between periods. For CRP levels (symbiotic: $3038 \cdot 1$ (SD 4847.6) to $10485 \cdot 7$ (SD 6676.2) nmol/1; control: $2933 \cdot 3$ (sD 3695.2) to $13571 \cdot 4$ (sD 9590.4) nmol/1 and global leucocyte counts (symbiotic: $8 \cdot 4$ (SD 2.9) to $11 \cdot 2$ $(\mathrm{sD} 3.1) \times 10^{9} /$; control: $8.7(\mathrm{sD} 3.0)$ to $\left.11.5(\mathrm{sD} 5.0) \times 10^{9} / 1\right)$, there was an increase between pre- and postoperative periods $(P<0.05)$, but no statistical difference between groups. Results are shown as means and standard deviations.

\section{Discussion}

Symbiotics are expected to have a positive impact on intestinal permeability, improving intestinal function and lowering postoperative complications. It has been postulated that they can inhibit the adherence and growth of pathogenic microorganisms through the production of immunomodulatory metabolites, antimicrobial substances and the formation of biofilms over the gut wall. Through a synergic effect, probiotics strains ferment prebiotics and produce SCFA that have immunomodulatory effects and suppress TNF- $\alpha$ production. Symbiotics can also induce the expression of the tight junction protein occludin, increasing the stability of tight junction proteins and intestinal barrier function ${ }^{(23)}$. Intestinal permeability can be negatively affected by metabolically stressful situations such as operations, some diseases, and nutritional status, and symbiotics can be used as potential modulators in this situation.

For head and neck cancers, surgical treatment is the primary option in most cases ${ }^{(24)}$. Patients with this type of cancer are at higher risk of postoperative complications, as they are usually malnourished, a condition known to directly impact on the immunologic system and the development of infection ${ }^{(25)}$. A high prevalence of malnutrition, about $60 \%(26)$, is usually shown in the head and neck cancer population, data similar to our findings. Most likely, this depleted nutritional status is related to food restriction due to mechanical obstruction and odynophagia $^{(27)}$. In addition, patients in this study had been diagnosed with cancer with an average of 3 months, ranging up to 24 months, which may have contributed to a worst nutritional status due to the longer food deprivation. However, there was no difference in the presence of malnutrition between the groups, which represents similar intestinal functionality, as, undernutrition can also alter intestinal permeability ${ }^{(28)}$, affecting a patient's intestinal health. The surgical procedure per se is another risk factor related to intestinal health.

Therefore, we analysed serum DAO concentrations, but found no differences between the symbiotic and control groups. Other authors who compared symbiotic use with a placebo in surgical patients also found no differences in permeability between groups $^{(29,30)}$. Moreover, intestinal function, complications of infection, and inflammatory and immunomodulation markers were not different between our study groups.

There was a higher incidence of fistula in the symbiotic group, but most occurred in the first $48 \mathrm{~h}$ and were thus usually related to technical issues. Therefore, the impact of symbiotic treatment on these fistulas should be interpreted with caution, as complications related to the surgical technique cannot be modified by nutritional treatment or the modulation of microbiota ${ }^{(31,32)}$.

Rammohan et $a l .{ }^{(8)}$, indicated that, contrary to our results, perioperative symbiotic administration was beneficial when compared with placebo in patients undergoing the Frey procedure for chronic pancreatitis. Patients received symbiotics orally three times daily, initiated $5 \mathrm{~d}$ preoperatively and up to $10 \mathrm{~d}$ after surgery. The infection rate was three times higher in the placebo group, with decreased antibiotic use and length of hospital stay in the symbiotic group ${ }^{(8)}$. A recent meta-analysis on the effects of perioperative probiotics and symbiotics on postoperative infections after gastrointestinal surgery also indicated beneficial effects of the administration of both probiotics and symbiotics ${ }^{(33)}$. It showed a reduction in postoperative infections, shorter lengths of hospital stay, and decreased antibiotic therapy for patients. 
Even after stratification of the studies assessing probiotics $v$. symbiotics, there were still beneficial results in both treatment modalities.

We hypothesise that several potential factors might explain why we obtained different results from those of the abovementioned studies. First, the administration of symbiotics in the postoperative period in our study might not have had an impact on microbiota homoeostasis, the mucosal barrier, or endothelial function because of the timing of administration. In most RCT with beneficial symbiotic effects, the treatment was initiated before the operation, thus showing a preventive role. Second, because head and neck procedures do not directly involve bowel manipulation, symbiotics may have had a restricted impact compared with symbiotics used in RTCs conducted in patients who underwent major abdominal surgery. Moreover, in our study, the symbiotic combination was fructooligosaccharides plus Lactobacillus acidophilus, L. rhamnosus, L. paracasei and Bifidobacterium lactis, in contrast to the most frequently used prebiotic in other studies that showed positive effects, galacto-oligosaccharides, and the most frequently used probiotic bacteria: L. plantarum, L. acidophilus, L. casei and $B$. breve. These considerable differences raise an important point: the results may differ because of the probiotic species recommendation. Some studies report that some probiotic supplements may not modify the microbiota profile, especially the lactobacillus strains ${ }^{(34-37)}$.

We analysed the bacterial profile by real-time PCR to identify potential pathogen colonisation (data not shown) that could explain some of the results. Although we found that Enterobacteriaceae species were increased after surgery in both the symbiotic and the control group, there was no statistical difference between them. Enterococcus species increased postoperatively in the symbiotic group, but this was also not statistically different from the numbers in the preoperative period or in the control group. These findings show there was an ongoing microbiota imbalance in these surgical patients despite interventions. Studies by Anderson et al. ${ }^{(13)}$ and McNaught et al. ${ }^{(38)}$, with different study populations but similar results to ours, indicate the need for more trials. Anderson et al. compared two groups, symbiotic (L. acidophilus, L. bulgaricus, B. lactis, S. thermophilus + oligofructose) and placebo, aiming to evaluate the impact of symbiotics on BT, systemic inflammation and postoperative complications in patients undergoing elective laparotomies and starting the intake of symbiotics $12 \mathrm{~d}$ before the operation until the fourth postoperative day. There were no differences between groups in BT, systemic inflammation, length of hospital stay, incidence of postoperative infections or mortality. McNaught et al. followed patients who underwent major elective abdominal surgery and who received a probiotic drink (L. plantarum) $9 \mathrm{~d}$ preoperatively and $5 \mathrm{~d}$ postoperatively and assessed the impact on BT and septic morbidity. The authors found no differences in the number of positive mesenteric lymph nodes between groups, nor in the CRP values or the incidence of septic morbidity.

Limitations of the current study may be related to the method used to assess intestinal permeability. DAO activity seems to be a good test to evaluate intestinal permeability in humans when compared with urinary sugar excretion tests, such as the lactulose-mannitol ratio test, or with mesenteric lymph node cultures (analyses that require a minimum of $6 \mathrm{~h}$ of urinary collection and a surgical procedure, respectively). However, the DAO activity test may not be the most sensitive for identifying alterations in intestinal permeability in surgical patients, as antibiotic use and aging may interfere with its activity ${ }^{(39,40)}$. In addition, our sample size was calculated to evaluate intestinal permeability, related to differences in DAO concentrations. Thus, this study was not statistically powered to compare groups in terms of complications and other assessed variables. Furthermore, it is important to address the postoperative administration of the symbiotics in which a shorter period of treatment may have been crucial to our results, in consideration of the mechanisms of action proposed to avoid postoperative complications $^{(1,23,41)}$. It seems that a preventive preoperative approach could help minimise the negative impact caused by the surgical stress on the intestinal barrier. Prebiotics, probiotics, and symbiotics seem to present better results when administered preoperatively.

\section{Conclusions}

Postoperative symbiotics did not impact on intestinal function and postoperative outcomes of head and neck surgical patients.

\section{Acknowledgements}

The authors would like to thank the head and neck surgery team at the Hospital das Clínicas da Universidade Federal de Minas Gerais for cooperation during the research and for helping the development of this project, specially professor José Maria Porcaro Salles, surgeons Alexandre de Andrade Sousa and Guilherme de Souza Silva, and the residente Lívio Bruno Santos Cunha. The authors also thank Invictus ${ }^{\circledR}$ Farmanutrition FQM Group for providing the study samples and Fundação de Amparo e Apoio à Pesquisa de Minas Gerais (FAPEMIG) for the financial support.

The authors contributed to all research phases, including the formulation of the research question, designing the study, carrying it out, data analysis and writing.

\section{References}

1. Correia MI, Liboredo JC \& Consoli ML (2012) The role of probiotics in gastrointestinal surgery. Nutrition $\mathbf{2 8}$, 230-234.

2. van Bokhorst-De van der Schuer MA, von Blomberg-van der Flier BM, Riezebos RK, et al. (1998) Differences in immune status between well-nourished and malnourished head and neck cancer patients. Clin Nutr 17, 107-111.

3. Gibson GR \& Roberfroid MB (1995) Dietary modulation of the human colonic microbiota: introducing the concept of prebiotics. J Nutr 125, 1401-1412.

4. Bazzocchi G, Giovannini T, Giussani C, Brigidi P \& Turroni S (2014) Effect of a new synbiotic supplement on symptoms, stool consistency, intestinal transit time and gut microbiota in patients with severe functional constipation: a pilot randomized double-blind, controlled trial. Tech Coloproctol 18, 945-953. 
5. Rayes N, Hansen S, Seehofer D, et al. (2002) Early enteral supply of fiber and lactobacilli versus conventional nutrition: a controlled trial in patients with major abdominal surgery. Nutrition 18, 609-615.

6. Rayes N, Seehofer D, Hansen S, et al. (2002) Early enteral supply of lactobacillus and fiber versus selective bowel decontamination: a controlled trial in liver transplant recipients. Transplantation 74, 123-127.

7. Rayes N, Seehofer D, Theruvath T, et al. (2005) Supply of preand probiotics reduces bacterial infection rates after liver transplantation-a randomized, double-blind trial. Am J Transplant 5, 125-130.

8. Rammohan A, Sathyanesan J, Rajendran K, et al. (2015) Synbiotics in surgery for chronic pancreatitis: are they truly effective? A single blind prospective randomized control trial. Ann Surg 262, 31-37.

9. Rayes N, Seehofer D, Theruvath T, et al. (2007) Effect of enteral nutrition and synbiotics on bacterial infection rates after pylorus-preserving pancreatoduodenectomy: a randomized, double-blind trial. Ann Surg 246, 36-41.

10. Tanaka K, Yano M, Motoori M, et al. (2012) Impact of perioperative administration of synbiotics in patients with esophageal cancer undergoing esophagectomy: a prospective randomized controlled trial. Surgery 152, 832-842.

11. Reddy BS, Macfie J, Gatt M, Larsen CN, Jensen SS \& Leser TD (2007) Randomized clinical trial of effect of synbiotics, neomycin and mechanical bowel preparation on intestinal barrier function in patients undergoing colectomy. Br J Surg 94, 546-554.

12. Krebs B (2016) Prebiotic and Synbiotic treatment before colorectal surgery - randomised double blind trial. Coll Antropol 40, 35-40.

13. Anderson AD, McNaught CE, Jain PK \& MacFie J (2004) Randomised clinical trial of synbiotic therapy in elective surgical patients. Gut 53, 241-245.

14. Bieganski T, Kusche J, Lorenz W, Hesterberg R, Stahlknecht CD \& Feussner KD (1983) Distribution and properties of human intestinal diamine oxidase and its relevance for the histamine catabolism. Biochim Biophys Acta 756, 196-203.

15. Takagi K, Nakao M, Ogura Y, Nabeshima T \& Kunii A (1994) Sensitive colorimetric assay of serum diamine oxidase. Clin Chim Acta 226, 67-75.

16. Edge SB \& Compton CC (2010) The American Joint Committee on Cancer: the 7th edition of the AJCC cancer staging manual and the future of TNM. Ann Surg Oncol 17, 1471-1474.

17. Detsky AS, McLaughlin JR, Baker JP, et al. (1987) What is subjective global assessment of nutritional status? JPEN J Parenter Enteral Nutr 11, 8-13.

18. Budziareck MB, Pureza Duarte RR \& Barbosa-Silva MC (2008) Reference values and determinants for handgrip strength in healthy subjects. Clin Nutr 27, 357-362.

19. Lewis SJ \& Heaton KW (1997) Stool form scale as a useful guide to intestinal transit time. Scand J Gastroenterol 32, 920-924.

20. Dindo D, Demartines N \& Clavien PA (2004) Classification of surgical complications: a new proposal with evaluation in a cohort of 6336 patients and results of a survey. Ann Surg $\mathbf{2 4 0}$, 205-213.

21. Kamiya S, Nagino M, Kanazawa H, et al. (2004) The value of bile replacement during external biliary drainage: an analysis of intestinal permeability, integrity, and microflora. Ann Surg 239, 510-517.

22. Armitage P \& Berry G (1987) The planning of statistical investigations. In Statistical Methods in Medical Research, 2nd ed., pp. 179-185 [P Armitage and G Berry, editors]. Oxford, UK: Blackwell Scientific.

23. Suchodolski JS \& Jergens AE (2016) Recent advances and understanding of using probiotic-based interventions to restore homeostasis of the microbiome for the prevention/ therapy of bacterial diseases. Microbiol Spectr 4, 1-14.

24. Galbiatti AL, Padovani-Junior JA, Maniglia JV, Rodrigues CD, Pavarino EC \& Goloni-Bertollo EM (2013) Head and neck cancer: causes, prevention and treatment. Braz J Otorhinolaryngol 79, 239-247.

25. van Bokhorst-de van der Schueren MA, van Leeuwen PA, Sauerwein HP, Kuik DJ, Snow GB \& Quak JJ (1997) Assessment of malnutrition parameters in head and neck cancer and their relation to postoperative complications. Head Neck 19, 419-425.

26. Jager-Wittenaar H, Dijkstra PU, Vissink A, van der Laan BF, van Oort RP \& Roodenburg JL (2011) Malnutrition and quality of life in patients treated for oral or oropharyngeal cancer. Head Neck 33, 490-496.

27. Gourin CG, Couch ME \& Johnson JT (2014) Effect of weight loss on short-term outcomes and costs of care after head and neck cancer surgery. Ann Otol Rhinol Laryngol 123, 101-110.

28. Takimoto Y, Yoshiuchi K, Shimodaira S \& Akabayashi A (2014) Diamine oxidase activity levels in anorexia nervosa. Int J Eat Disord 47, 203-205.

29. Sugawara G, Nagino M, Nishio H, et al. (2006) Perioperative synbiotic treatment to prevent postoperative infectious complications in biliary cancer surgery: a randomized controlled trial. Ann Surg 244, 706-714.

30. Kanazawa H, Nagino M, Kamiya S, et al. (2005) Synbiotics reduce postoperative infectious complications: a randomized controlled trial in biliary cancer patients undergoing hepatectomy. Langenbecks Arch Surg 390, 104-113.

31. Levine EA \& Alverdy JC (1990) Carotid-esophageal fistula following a penetrating neck injury: case report. J Trauma $\mathbf{3 0}$, $1588-1590$

32. Shogan BD, Smith DP, Christley S, Gilbert JA, Zaborina O \& Alverdy JC (2014) Intestinal anastomotic injury alters spatially defined microbiome composition and function. Microbiome $\mathbf{2}, 35$.

33. Yang Z, Wu Q, Liu Y \& Fan D (2017) Effect of perioperative probiotics and synbiotics on postoperative infections after gastrointestinal surgery: a systematic review with metaanalysis. JPEN J Parenter Enteral Nutr 41, 1051-1062.

34. Wang R, Chen S, Jin J, et al. (2015) Survival of Lactobacillus casei strain Shirota in the intestines of healthy Chinese adults. Microbiol Immunol 59, 268-276.

35. Stadlbauer V, Leber B, Lemesch S, et al. (2015) Lactobacillus casei shirota supplementation does not restore gut microbiota composition and gut barrier in metabolic syndrome: a randomized pilot study. PLOS ONE 10, e0141399.

36. Kristensen NB, Bryrup T, Allin KH, Nielsen T, Hansen TH \& Pedersen O (2016) Alterations in fecal microbiota composition by probiotic supplementation in healthy adults: a systematic review of randomized controlled trials. Genome Med $\mathbf{8}, 52$.

37. Gorshein E, Wei C, Ambrosy S, et al. (2017) Lactobacillus rhamnosus GG probiotic enteric regimen does not appreciably alter the gut microbiome or provide protection against GVHD after allogeneic hematopoietic stem cell transplantation. Clin Transplant 31, 1-15.

38. McNaught CE, Woodcock NP, MacFie J \& Mitchell CJ (2002) A prospective randomised study of the probiotic Lactobacillus plantarum $299 \mathrm{~V}$ on indices of gut barrier function in elective surgical patients. Gut 51, 827-831.

39. Maintz L \& Novak N (2007) Histamine and histamine intolerance. Am J Clin Nutr 85, 1185-1196.

40. Xie Q \& Gan HT (2010) Controversies about the use of serological markers in diagnosis of inflammatory bowel disease. World J Gastroenterol 16, 279-280.

41. Jeppsson B, Mangell P \& Thorlacius H (2011) Use of probiotics as prophylaxis for postoperative infections. Nutrients $\mathbf{3}$, 604-612. 